\title{
A Comparative Application for Evaluating Composite Fabrics Used in Electromagnetic Shielding
}

\author{
F. Gamze Kizilcay Abdulla \\ Electrical Electronics Engineering \\ Engineering Faculty \\ Yuzuncu Yil University \\ Van, Turkey \\ gamzekizilcay@yyu.edu.tr
}

\author{
Ruslan Abdulla \\ Computer Technologies T.O.B.B. T.S. Vocational School \\ Karabuk University \\ Karabuk, Turkey \\ ruslanabdulla@karabuk.edu.tr
}

\begin{abstract}
Composite fabrics containing metal filaments are used widely for preventing electromagnetic radiation. Many experiments involving them are carried out continuously. Results are simulated in order to analyze their performance. Coding in Matlab is a popular method to compare the electromagnetic shielding properties of composite fabrics but for different options Matlab codes must be edited each time. Scientists who are not experts in coding have difficulties on editing such codes. To overcome this, an application written in C\# in Visual Studio with .Net platform was developed. This application is integrated with Bunifu, which allows the application to be well designed. The proposed interface is user friendly and lets the user choose the available fabric with its stitch length from panel section. By setting options one can get the electromagnetic parameters such as scattering ( $(S)$ parameters, reflection, transmission and absorption coefficients and total shielding effectiveness (SE) values with one click. As the application is integrated with Matlab codes, output is given as a Matlab graph with desired options. In this way the distinctions between the chosen fabrics can be analyzed easily.
\end{abstract}

Keywords-Composite fabric; electromagnetic shielding; textile application; user interface

\section{INTRODUCTION}

Electronic devices cause electromagnetic pollution via irradiation. In such situations the body can not condemn its temperature and invites illnesses [1-3]. The most common devices that cause daily exposure at home are mobile phones and deck phones operating in RF and microwave frequencies, DSL and WiFi modems, AM signals and satellite broadcasts, microwave ovens, etc. [3-4]. Many methods have been developed for obstructing electromagnetic radiation. One of them is shielding. Shielding is defined as isolating the two media at circuit or device level in an electromagnetic sense. Thus, a shielding material blocks completely or partially the passage of the signal. As shielding material, conductive or ferromagnetic materials are usually used. The conductance or magnetic permeability of these materials plays an active role in the reflection or absorption of the electromagnetic signal [5]. Protective textile fabrics are commonly seen as shielding materials in electromagnetic shielding applications. These fabrics are known as conductive fabrics because the yarns that make up the fabric consist of fine metal filaments blended with the yarns. The main characteristic of conductive fabric is the ability to use conductive or ferromagnetic materials in its structure as a shield for electromagnetic fields. Their main advantage is that they are flexible and therefore can cover any kind of gap and can prevent leaks from connection points. Ease of mass production and low cost are also advantages of conductive fabrics [6-7]. The use area of conductive fabrics is quite wide. Today this industrialized area can be seen in wearable technology, military and health [8].

A network analyzer is often used in electromagnetic shielding experiments. This device generates scattering parameter values which give clues about the shielding performance of the shielding material. It is possible to obtain reflection, transmission and absorption coefficient values as well as total electromagnetic shielding values by using appropriate conversion formulas from scattering parameters. With this application, scattering parameters and other electromagnetic units are displayed in options section. The scattering parameters obtained from electromagnetic shielding experiments of copper, silver and stainless steel cored cardigan, lacoste, milano, rib and weft knitted fabrics were used in this study. The application has an interface that allows comparison between the shielding materials displayed on main panel. The display of electromagnetic shielding results is usually done with Matlab graphics. This requires the user to be familiar with Matlab codes. However, it is still possible to develop an interface using the Matlab gui features suitable for the user. But still, it is not aesthetic to make this interface with Matlab and there are some deficiencies in reading the stored data from the computer as compared to the $\mathrm{c \#}$ language. For this reason, in this work c\# code is used on the Visual Studio .Net platform. It is quite possible to develop better interface on the .Net platform. This is even easier with a plugin named Bunifu. By integrating Bunifu it is much easier to get aesthetic designs, as interface objects can use the same $\mathrm{c} \#$ code properties and methods. 


\section{APPLICATION}

This desktop application is made by using Matlab and visual studio application programming interface (API). The functional part of the program is implemented with these two API's. With Matlab, conversion formulas are executed according to user's preferences and results are displayed with graphs. With Visual Studio, easy interface is provided for the user and preferences are sent to Matlab environment for processing. A Bunifu plugin with its well designed objects was integrated with visual studio for a better interface.

\section{A. Interface}

When the application is opened, two panels are displayed showing the fabric name and stitch length. This information is automatically retrieved from the application's database (Figure 1). These lists are automatically updated when different fabrics are added to or removed from the database. It is seen that the program page is widened by clicking on the stitch density of that fabric whichever fabric is desired to be compared (Figure 2). In the expanding section, the selected fabric, stitch length and metal filament type are seen. In this example, copper cored lacoste, milano, cardigan, rib and weft knitted fabrics with a $4 \mathrm{~mm}$ stitch density are selected. In the right part of the expanding section, there are options of electromagnetic parameters and graphical view. In the options section, one can select S parameters, and accordingly any of the options of reflection, transmission, absorption coefficients and total shielding effectiveness. Preferences are configured so that only one electromagnetic parameter can be evaluated at a time. Features other than electromagnetic parameters such as grid and markers relate to Matlab graphical interface. It is recommended that these properties are set on for more precise analysis.

\section{B. Working Principle}

After running the application the user interface loads. During the loading process, required Matlab packages and Bunifu plugin load and are getting ready to execute. Afterwards the data available are extracted into the interface. In the second stage, user chooses a fabric and stitch length. Accordingly he decides which fabrics' electromagnetic properties are going to be compared. The user can select or deselect the fabric. After choosing the fabric user decides which graphics should be displayed. In the application presented electromagnetic shielding parameter options are scattering parameters, reflection, transmission and absorption parameters and the shielding effectiveness magnitudes. There are also minor properties about the viewing graphics. There are grids and markers which could be set on or off. The workflow is shown in Figure 3.

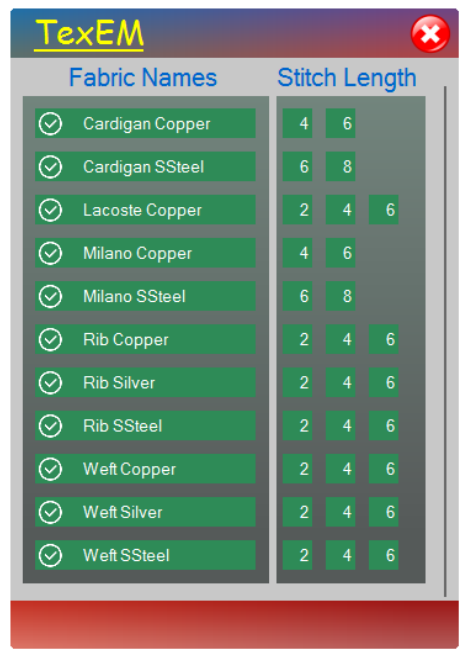

Fig. 1. User interface

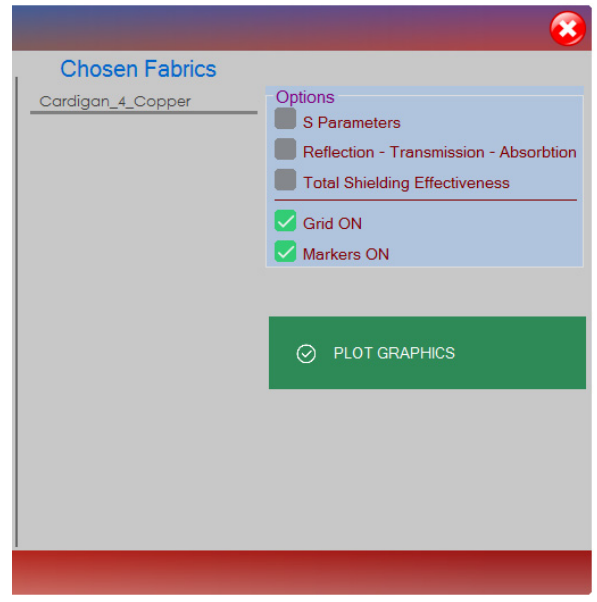

Fig. 2. Expanding section

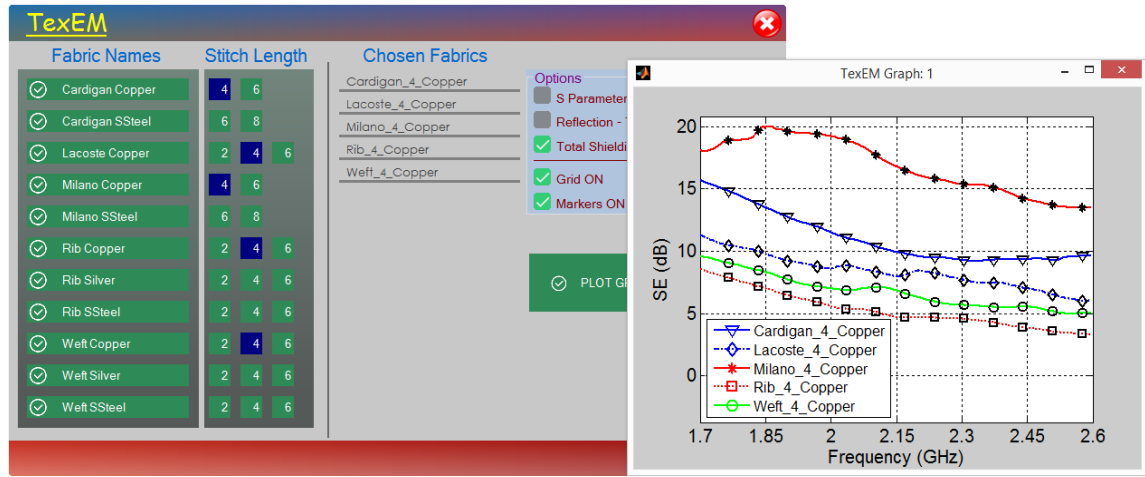

Fig. 3. An ordinary output (SE) due user's preferences. 


\section{Output}

Electromagnetic parameters provided by the application are reachable on the options section. Specific results are illustrated upon users' preferences (Figure 4). This can be thought of as the best example of the compatibility of the interface with the graphical environment. It is also possible to obtain other electromagnetic parameters with the same shielding material. The relevant results are shown in graphics in Appendix.

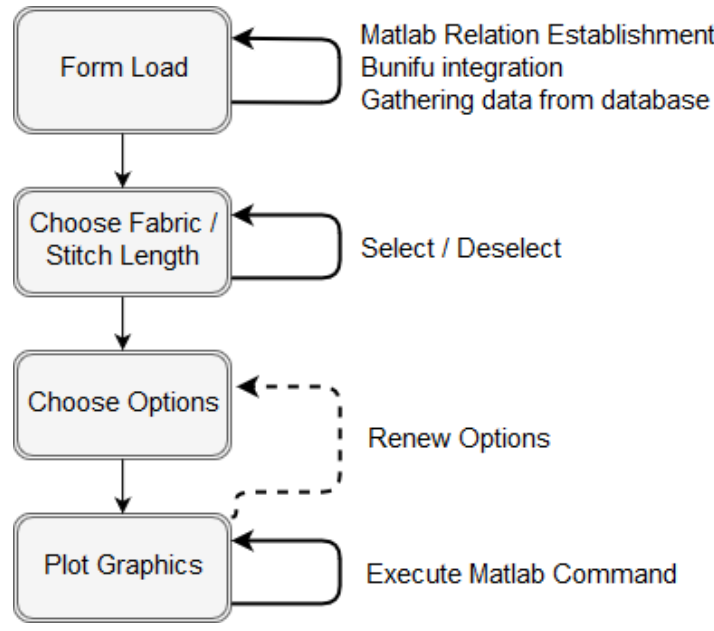

Fig. 4. Working principle of application.

\section{CONCLUSION}

The application developed within the scope of this study has user friendly and functional features to meet the needs of researchers who are interested in the effects of electromagnetic shielding of textile fabrics but have difficulties in coding. Automatic extraction of fabric information from the database makes the application dynamic and sustainable. When different fabrics are desired to be compared, entry of the relevant test results into the database is sufficient for the operation of the application. The results are shown graphically in Matlab environment. In this way, the user can compare and analyze the electromagnetic shielding parameters of the desired fabrics, such as $\mathrm{S}$, reflection, transmission, absorption parameters and total shielding effectiveness values. Besides the introduction of new knitted fabrics to the proposed application, the addition and classification of woven conductive fabrics used in electromagnetic shielding, saving preferences, extracting statistics from graphs, adding visual themes can be considered as features for further studies. We think that the proposed study and application will have a positive impact on the literature.

\section{REFERENCES}

[1] T. E. Aldrich, C. E. Easterly, "Electromagnetic Fields and Public Health", Environmental Health Perspectives, Vol. 75, pp. 159-171,1987

[2] V. De Santis, "Ear Temperature Increase Produced by Cellular Phones Under Extreme Exposure Conditions", IEEE Transactions on Microwave Theory and Techniques, Vol. 60, No. 6, pp. 1728-1734, 2012

[3] L. Cheng, T. Zhang, M. Guo, J. Li, S. Wang, H. Tang, "Electromagnetic shielding effectiveness and mathematical model of stainless steel composite fabric", The Journal of The Textile Institute. Vol. 106, No. 6, pp. $577-586,2014$

[4] H. Ozdemir, S. S. Ugurlu, A. Ozkurt, "The electromagnetic shielding of textured steel yarn based woven fabrics used for clothing", Journal of Industrial Textiles, Vol. 45, No. 3, pp. 416-436, 2015

[5] L. Sevgi, Elektromanyetik Uyumluluk Elektromanyetik Kirlilik, Yapım Matbaa, 2000

[6] B. S. Dasaradan, "Electromagnetic Shielding Effectiveness of Copper Core Yarn Knitted Fabrics", Indian Journal of Fibre and Textile Research, Vol. 34, pp. 149-154, 2009

[7] Z. Liu, X. C. Wang, "Influence of fabric weave type on the effectiveness of electromagnetic shielding woven fabric", Journal of Electromagnetic Waves and Applications, Vol. 26, No. 14-15, pp. 1848-1856, 2012

[8] J. Banaszczyk, G. De Mey, A. Schwarz, L. Van Langenhove, "Current Distribution Modelling in Electrocundtive Fabrics", Fibres \& Textiles in Eastern Europe, Vol. 17, No. 2 (73), pp. 28-33, 2009

APPENDIX

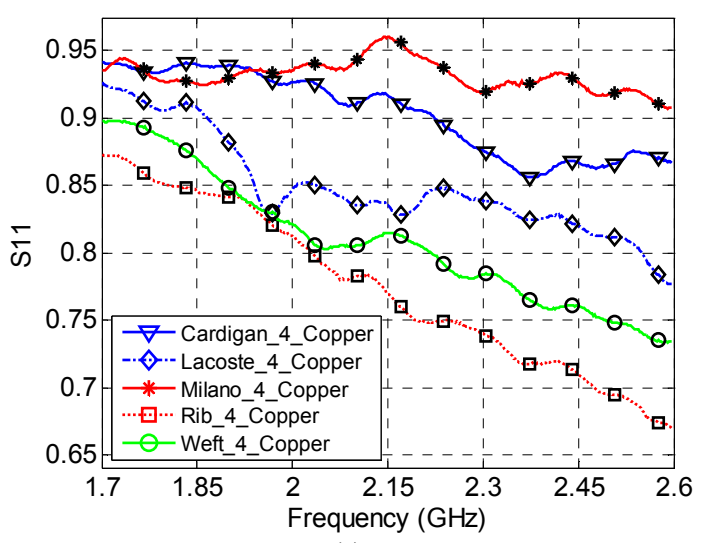

(a)

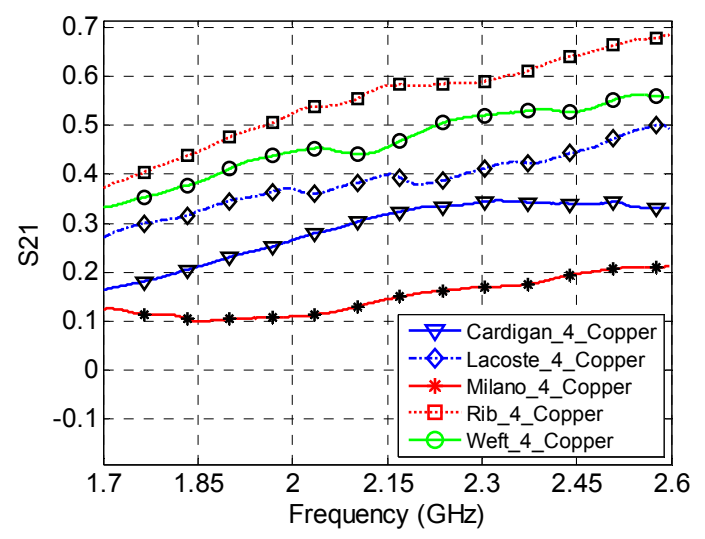

(b)

Fig. A1.

Scattering parameters a) S11 b) S21. 


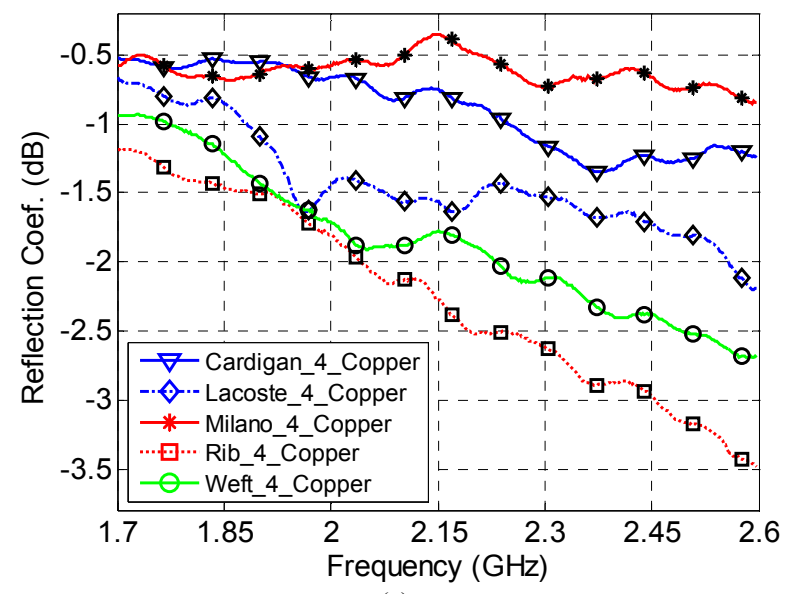

(a)

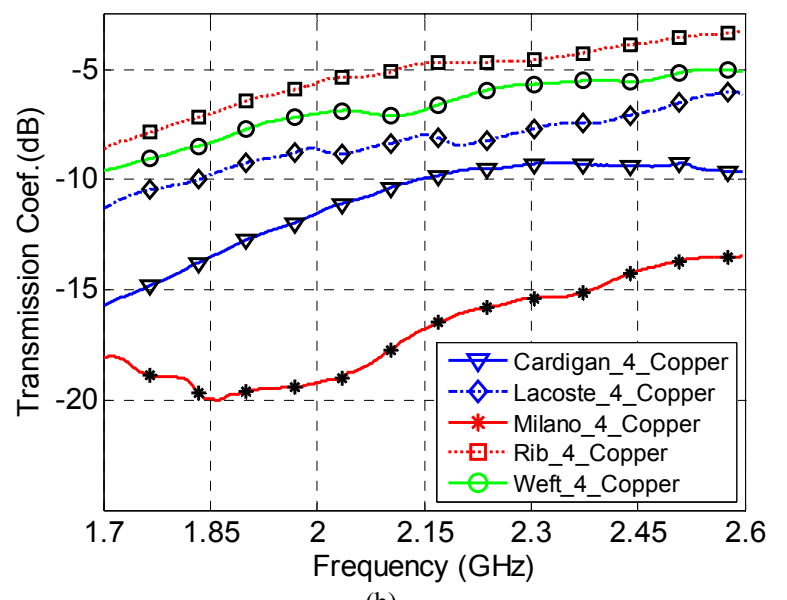

(b)

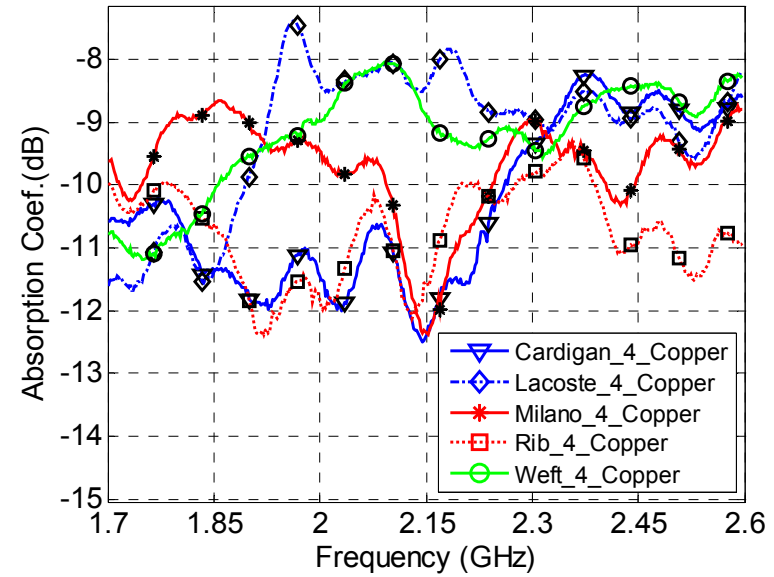

(c)

Fig. A2. Coefficients a) Reflection b) Transmission c) Absorption. 